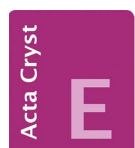

CRYSTALLOGRAPHIC COMMUNICATIONS

ISSN 2056-9890

Received 3 October 2016

Accepted 15 October 2016

Edited by M. Weil, Vienna University of Technology, Austria

Keywords: crystal structure; uranium(V); nitrate; ammonia; hydrogen bonding.

CCDC reference: 1510182

Supporting information: this article has supporting information at journals.iucr.org/e

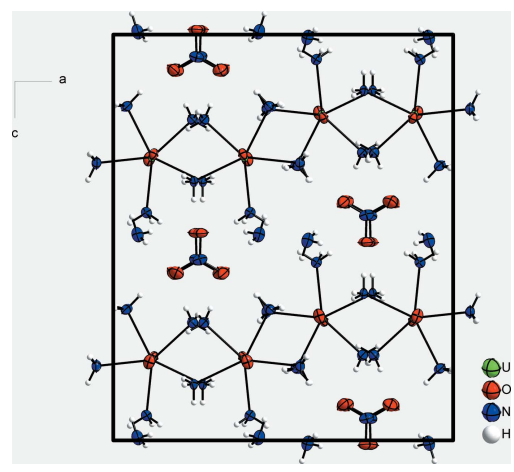

OPEN $\odot$ ACCESS

\section{Crystal structure of $\left[\mathrm{UO}_{2}\left(\mathrm{NH}_{3}\right)_{5}\right] \mathrm{NO}_{3} \cdot \mathrm{NH}_{3}$}

\author{
Patrick Woidy and Florian Kraus*
}

Anorganische Chemie, Fluorchemie, Philipps-Universität Marburg, Hans-Meerwein-Strasse 4, 35032 Marburg, Germany.
*Correspondence e-mail: florian.kraus@chemie.uni-marburg.de

Pentaammine dioxide uranium(V) nitrate ammonia (1/1), $\left[\mathrm{UO}_{2}\left(\mathrm{NH}_{3}\right)_{5}\right] \mathrm{NO}_{3}$.$\mathrm{NH}_{3}$, was obtained in the form of yellow crystals from the reaction of caesium uranyl nitrate, $\mathrm{Cs}\left[\mathrm{UO}_{2}\left(\mathrm{NO}_{3}\right)_{3}\right]$, and uranium tetrafluoride, $\mathrm{UF}_{4}$, in dry liquid ammonia. The $\left[\mathrm{UO}_{2}\right]^{+}$cation is coordinated by five ammine ligands. The resulting $\left[\mathrm{UO}_{2}\left(\mathrm{NH}_{3}\right)_{5}\right]$ coordination polyhedron is best described as a pentagonal bipyramid with the $\mathrm{O}$ atoms forming the apices. In the crystal, numerous $\mathrm{N}-\mathrm{H} \cdots \mathrm{N}$ and $\mathrm{N}-\mathrm{H} \cdots \mathrm{O}$ hydrogen bonds are present between the cation, anion and solvent molecules, leading to a three-dimensional network.

\section{Introduction - Chemical context}

Uranium chemistry in aqueous solution is dominated by the uranyl cation $\left[\mathrm{UO}_{2}\right]^{2+}$, with the uranium atom in the hexavalent oxidation state. The most prominent representatives are the well-known uranyl nitrates and uranyl halides. In contrast to the $\left[\mathrm{UO}_{2}\right]^{2+}$ uranyl cation, the uranyl cation $\left[\mathrm{UO}_{2}\right]^{+}$ with pentavalent uranium disproportionates in aqueous solution into the $\left[\mathrm{U}^{\mathrm{VI}} \mathrm{O}_{2}\right]^{2+}$ cation and a tetravalent uranium species. Only under controlled conditions (Kraus et al., 1949) and in organic solvents (Arnold et al., 2009) are uranyl cations with pentavalent uranium observable. Here we report on the crystal structure of a $\mathrm{U}^{\mathrm{V}}$ compound, $\left[\mathrm{UO}_{2}\left(\mathrm{NH}_{3}\right)_{5}\right] \mathrm{NO}_{3} \cdot \mathrm{NH}_{3}$, obtained from the reaction of $\mathrm{U}^{\mathrm{IV}}$ with $\mathrm{U}^{\mathrm{VI}}$ species in anhydrous liquid ammonia. The compound is not stable at temperatures above $c a 238 \mathrm{~K}$ due to the loss of ammonia of a still unknown amount. Despite several efforts, we have not yet been able to reproduce the synthesis of the compound.

Obviously, the two uranium compounds used as educts reacted in a comproportionation reaction in order to form the $\mathrm{U}^{\mathrm{V}}$ compound reported here. It is possible that the redox potentials in liquid ammonia are reversed compared to aqueous solutions, leading to a comproportionation. Such changes of electrochemical potentials are not uncommon and, for example, are known for the system $\mathrm{Cu} / \mathrm{Cu}^{+} / \mathrm{Cu}^{2+}$ (Woidy et al., 2015a). However, the detailed reaction $\mathrm{U}^{\mathrm{VI}}+\mathrm{U}^{\mathrm{IV}} \rightarrow \mathrm{U}^{\mathrm{V}}$ is still unclear, and despite some efforts we were not able to elucidate further reaction products which must be present (e.g. fluoride containing ones).

\section{Results and discussion - Structural commentary}

All atoms in the structure of the title compound reside on general Wyckoff positions $8 c$ of space group Pbca. The pentavalent uranium atom $\mathrm{U} 1$ and the oxygen atoms $\mathrm{O} 1$ and $\mathrm{O} 2$ form an uranyl cation. This $\left[\mathrm{UO}_{2}\right]^{+}$ion is coordinated by five ammine ligands (N1-N5) forming the complex pentagonal-bipyramidal $\left[\mathrm{UO}_{2}\left(\mathrm{NH}_{3}\right)_{5}\right]^{+}$cation which is shown in 


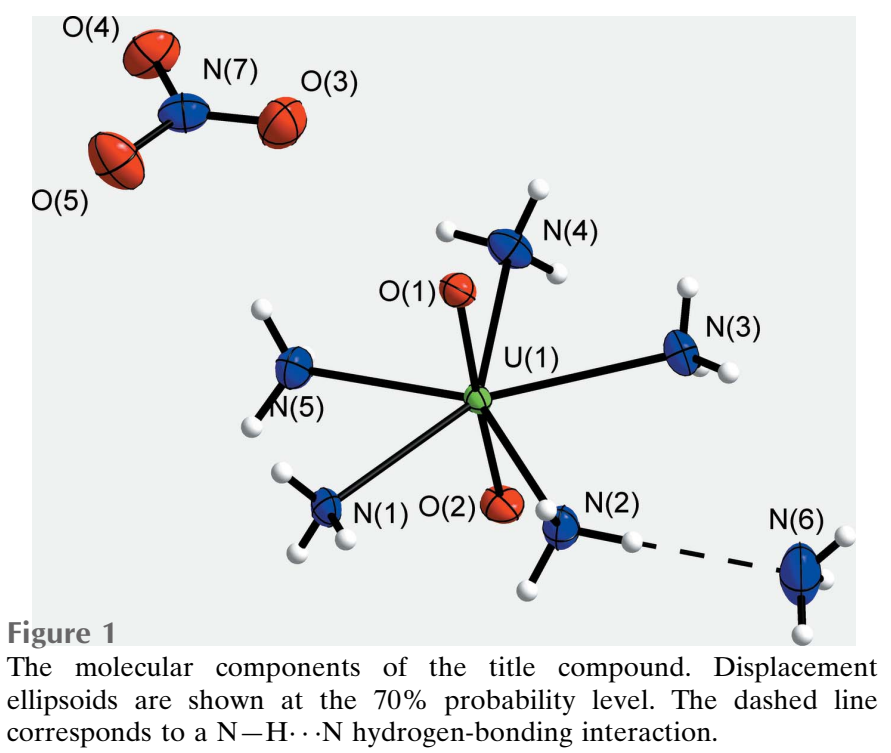

Fig. 1. The nitrate anion $\mathrm{NO}_{3}{ }^{-}$consists of the nitrogen atom $\mathrm{N} 7$ and the oxygen atoms O3-O5. An ammonia molecule of crystallization (N6) is also observed in the structure.

The $\mathrm{U}-\mathrm{O}$ distances in the almost linear uranyl cation [O$\mathrm{U}-\mathrm{O}$ angle of $\left.177.2(1)^{\circ}\right]$ are $1.861(3)$ and $1.867(3) \AA$, respectively. Such distances are slightly elongated compared to reported ones for uranyl compounds with pentavalent uranium (Berthet et al., 2003; Hayton \& Wu, 2008), which are in the range 1.810 (4) to 1.828 (4) $\AA$. However, U-O distances for uranyl cations $\left[\mathrm{UO}_{2}\right]^{2+}$ with hexavalent uranium are about 0.02 to $0.07 \AA$ shorter. For the alkali metal uranyl nitrates, such as $M\left[\mathrm{UO}_{2}\left(\mathrm{NO}_{3}\right)_{3}\right]$ with $M=\mathrm{K}$ (Jouffret et al., 2011; Krivovichev \& Burns, 2004), Rb (Barclay et al., 1965; Zalkin et al., 1989) and Cs (Malcic \& Ljubica, 1961), the reported $\mathrm{U}-\mathrm{O}$ distances are in the range 1.746 to $1.795 \AA$. In uranium(VI) compounds that contain the comparable pentaammine dioxido uranium(VI) ion $\left[\mathrm{UO}_{2}\left(\mathrm{NH}_{3}\right)_{5}\right]^{2+}$, such as $\left[\mathrm{UO}_{2}\left(\mathrm{NH}_{3}\right)_{5}\right] \mathrm{Cl}_{2} \cdot \mathrm{NH}_{3}, \quad\left[\mathrm{UO}_{2} \mathrm{~F}_{2}\left(\mathrm{NH}_{3}\right)_{3}\right]_{2} \cdot 2 \mathrm{NH}_{3}$ or $\left[\mathrm{UO}_{2}\left(\mathrm{NH}_{3}\right)_{5}\right] \mathrm{Br}_{2} \cdot \mathrm{NH}_{3}, \mathrm{U}-\mathrm{O}$ distances in the range 1.768 (2) to 1.771 (3) $\AA$ were reported (Woidy et al., 2012, 2015b); these are shortened by $c a 0.1 \AA$ compared to the uranyl ion presented here.

The nitrogen atoms of the ammine ligands show $\mathrm{U}-\mathrm{N}$ distances between 2.573 (3) and 2.629 (3) $\AA$, which appear slightly elongated in comparison with the $\mathrm{U}-\mathrm{N}$ distances determined for $\mathrm{U}^{\mathrm{VI}}$ compounds such as $\left[\mathrm{UO}_{2}\left(\mathrm{NH}_{3}\right)_{5}\right] \mathrm{Cl}_{2} \cdot \mathrm{NH}_{3}$ [2.505 (2)-2.554 (3) $\AA], \quad\left[\mathrm{UO}_{2}\left(\mathrm{NH}_{3}\right)_{5}\right] \mathrm{Br}_{2} \cdot \mathrm{NH}_{3} \quad$ or $\left[\mathrm{UO}_{2} \mathrm{~F}_{2}\left(\mathrm{NH}_{3}\right)_{3}\right]_{2} \cdot 2 \mathrm{NH}_{3}[2.522$ (3) to 2.577 (3) $\AA$ ] (Woidy et al., 2012). In $\left[\mathrm{UF}_{4}\left(\mathrm{NH}_{3}\right)_{4}\right] \cdot \mathrm{NH}_{3}$ (Kraus \& Baer, 2009), we observed an elongated $\mathrm{U}-\mathrm{N}$ distance of 2.618 (5) $\AA$ due to the higher coordination number and different charge of the central atom.

The nitrate anion features no unexpected structural parameters and is practically identical compared to the nitrate anions of $\mathrm{NaNO}_{3}$ or $\mathrm{KNO}_{3}$. The $\mathrm{N}-\mathrm{O}$ distances are 1.242 (5), 1.253 (4), and 1.254 (4) $\AA$, the bond angles are $120^{\circ}$ within the $3 \sigma$ criterion $\left[120.4(3), 120.4(3)\right.$, and $\left.119.2(3)^{\circ}\right]$ and therefore the anion is essentially planar.
Table 1

Hydrogen-bond geometry $\left(\AA,^{\circ}\right)$.

\begin{tabular}{lllll}
\hline$D-\mathrm{H} \cdots A$ & $D-\mathrm{H}$ & $\mathrm{H} \cdots A$ & $D \cdots A$ & $D-\mathrm{H} \cdots A$ \\
\hline $\mathrm{N} 1-\mathrm{H} 1 A \cdots \mathrm{O} 4^{\mathrm{i}}$ & 0.91 & 2.43 & $3.166(4)$ & 138 \\
$\mathrm{~N} 1-\mathrm{H} 1 A \cdots \mathrm{O} 4^{\mathrm{ii}}$ & 0.91 & 2.47 & $2.996(4)$ & 117 \\
$\mathrm{~N} 1-\mathrm{H} 1 B \cdots 1^{\mathrm{iii}}$ & 0.91 & 2.25 & $3.079(4)$ & 151 \\
$\mathrm{~N} 1-\mathrm{H} 1 C \cdots 2^{\mathrm{iv}}$ & 0.91 & 2.12 & $3.006(4)$ & 165 \\
$\mathrm{~N} 2-\mathrm{H} 2 A \cdots \mathrm{O} 4^{\mathrm{i}}$ & 0.91 & 2.49 & $3.220(5)$ & 138 \\
$\mathrm{~N} 2-\mathrm{H} 2 B \cdots \mathrm{N} 6$ & 0.91 & 2.14 & $3.024(5)$ & 164 \\
$\mathrm{~N} 2-\mathrm{H} 2 C \cdots 4^{\mathrm{ii}}$ & 0.91 & 2.36 & $3.232(5)$ & 160 \\
$\mathrm{~N} 3-\mathrm{H} 3 A \cdots 2^{\mathrm{v}}$ & 0.91 & 2.27 & $3.136(5)$ & 159 \\
$\mathrm{~N} 3-\mathrm{H} 3 B \cdots 1^{\mathrm{vi}}$ & 0.91 & 2.34 & $3.151(4)$ & 149 \\
$\mathrm{~N} 3-\mathrm{H} 3 C \cdots 5^{\mathrm{vii}}$ & 0.91 & 2.52 & $3.142(5)$ & 126 \\
$\mathrm{~N} 4-\mathrm{H} 4 A \cdots \mathrm{O} 1^{\mathrm{vi}}$ & 0.91 & 2.37 & $3.219(4)$ & 156 \\
$\mathrm{~N} 4-\mathrm{H} 4 B \cdots 2^{\mathrm{v}}$ & 0.91 & 2.26 & $3.086(4)$ & 150 \\
$\mathrm{~N} 4-\mathrm{H} 4 C \cdots \mathrm{O} 3$ & 0.91 & 2.55 & $3.253(5)$ & 134 \\
$\mathrm{~N} 5-\mathrm{H} 5 A \cdots \mathrm{O} 5^{\mathrm{iii}}$ & 0.91 & 2.14 & $3.048(5)$ & 176 \\
$\mathrm{~N} 5-\mathrm{H} 5 B \cdots \mathrm{O} 3$ & 0.91 & 2.44 & $3.063(5)$ & 126 \\
$\mathrm{~N} 5-\mathrm{H} 5 B \cdots \mathrm{O} 5$ & 0.91 & 2.59 & $3.394(5)$ & 147 \\
$\mathrm{~N} 5-\mathrm{H} 5 C \cdots 1^{\mathrm{iii}}$ & 0.91 & 2.37 & $3.273(4)$ & 171 \\
$\mathrm{~N} 6-\mathrm{H} 6 A \cdots \mathrm{O} 4^{\mathrm{vii}}$ & $0.86(7)$ & $2.50(7)$ & $3.342(6)$ & $167(7)$ \\
$\mathrm{N} 6-\mathrm{H} 6 B \cdots 3^{\mathrm{vi}}$ & $0.81(8)$ & $2.32(8)$ & $3.102(6)$ & $162(7)$ \\
& & & &
\end{tabular}

Symmetry codes: (i) $-x+\frac{1}{2},-y, z+\frac{1}{2}$; (ii) $x,-y+\frac{1}{2}, z+\frac{1}{2}$; (iii) $-x+\frac{1}{2}, y+\frac{1}{2}, z$; (iv) $-x+\frac{1}{2}, y-\frac{1}{2}, z ;$ (v) $-x, y-\frac{1}{2},-z+\frac{3}{2}$; (vi) $-x, y+\frac{1}{2},-z+\frac{3}{2}$; (vii) $x-\frac{1}{2}, y,-z+\frac{3}{2}$.

As we are not able to completely explain the formation of the title compound from the educts, the question arises whether the cation is not simply a 'regular' uranyl(VI) cation. It is obvious that no second nitrate anion is present in the structure. Due to chemical reasoning, the ammonia molecule of crystallization also cannot be an amide anion $\left(\mathrm{NH}_{2}{ }^{-}\right)$. As ammine ligands are bound to the uranium cation, some of their electron density is transferred to the Lewis-acidic $\mathrm{U}$ atom, which leads to a weakening of the $\mathrm{N}-\mathrm{H}$ bonds and therefore to an acidification of these protons. So, an amide anion residing next to an acidified ammine ligand is not a plausible assumption, especially since the ammonia molecule of crystallization shows an usual $\mathrm{N} \cdots \mathrm{N}$ distance for $\mathrm{N}-\mathrm{H} \cdots \mathrm{N}$ hydrogen bonds. If one assumes that $\mathrm{CO}_{3}{ }^{2-}$ is present instead of $\mathrm{NO}_{3}{ }^{-}$, then a 'regular' $\left[\mathrm{U}^{\mathrm{VI}} \mathrm{O}_{2}\right]^{2+}$ ion would also result. However, if one refines the occupancy of the $\mathrm{N}$ atom of the nitrate anion, an occupancy of 1.00 (2) is observed, whereas if the occupancy of the $\mathrm{C}$ atom of a putative carbonate anion is refined, an occupancy of 1.30 (2) is obtained. Comparing the atomic distances of the trigonal-planar anion with the mean distances from the literature, $1.284 \AA$ for $\mathrm{CO}_{3}{ }^{2-}$ (Zemann, 1981) and $1.250 \AA$ for $\mathrm{NO}_{3}{ }^{-}$(Baur, 1981), it is most likely that in our case a nitrate anion is present. In summary, all these points indicate that the central atom is an $\mathrm{N}$ atom of a nitrate anion. Together with the observation of slightly elongated $\mathrm{U}-$ $\mathrm{O}$ and $\mathrm{U}-\mathrm{N}$ bond lengths in comparison to similar $\left[\mathrm{UO}_{2}\left(\mathrm{NH}_{3}\right)_{5}\right]^{2+}$ ions, we conclude that the compound should contain $\mathrm{U}^{\mathrm{V}}$ atoms in form of $\left[\mathrm{UO}_{2}\right]^{+}$ions.

\section{Supramolecular features}

The crystal structure of the title compound is shown in Fig. 2. The ammonia molecule of crystallization (N6) acts as an acceptor of an $\mathrm{N}-\mathrm{H}$ hydrogen bond with an ammine ligand (N2). It forms also two disparate $\mathrm{N}-\mathrm{H} \cdots \mathrm{O}$ hydrogen bonds 


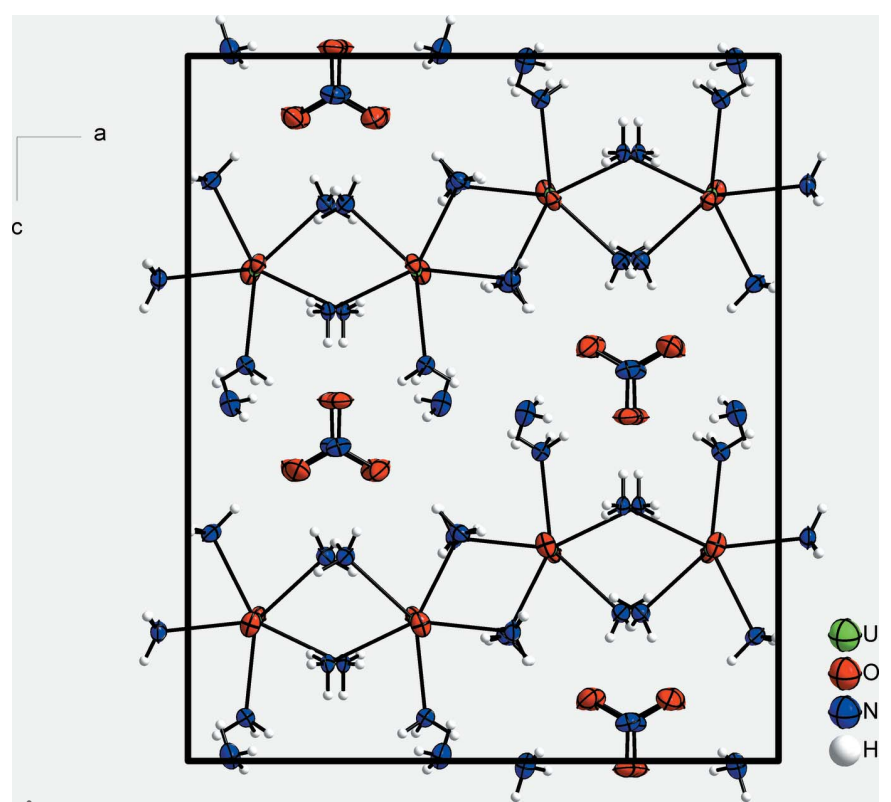

Figure 2

Crystal structure of $\left[\mathrm{UO}_{2}\left(\mathrm{NH}_{3}\right)_{5}\right] \mathrm{NO}_{3} \cdot \mathrm{NH}_{3}$ viewed along [010]. Displacement ellipsoids are shown at the $70 \%$ probability level.

to two symmetry-equivalent nitrate anions; the third $\mathrm{H}$ atom $(\mathrm{H} 6 \mathrm{C})$ is not involved in hydrogen-bond formation. The nitrate anion is hydrogen-bonded to five symmetry-related $\left[\mathrm{UO}_{2}\left(\mathrm{NH}_{3}\right)_{5}\right]^{+}$cations via $\mathrm{N}-\mathrm{H} \cdots \mathrm{O}$ hydrogen bonds and two symmetry-related ammonia molecules of crystallization. The nitrate anions lie parallel to the $a c$ plane and are arranged in columns running parallel to the $b$ axis (Fig. 2). The oxygen atoms of the uranyl cation act as acceptors of hydrogen bonds from four (O1) and three $(\mathrm{O} 2)$ ammine ligands of two symmetry-related $\left[\mathrm{UO}_{2}\left(\mathrm{NH}_{3}\right)_{5}\right]^{+}$cations. The linear $\mathrm{UO}_{2}{ }^{+}$ cations are also arranged parallel to the $b$ axis. Overall, a three-dimensional hydrogen-bonded network results. Numerical details of the hydrogen bonding interactions are compiled in Table 1.

\section{Synthesis and crystallization}

The purity of the used educts was evidenced by powder X-ray diffraction and IR spectroscopy. $50 \mathrm{mg}$ (0.09 mmol, 1 eq.) $\mathrm{Cs}\left[\mathrm{UO}_{2}\left(\mathrm{NO}_{3}\right)_{3}\right]$ and $27 \mathrm{mg}(0.09 \mathrm{mmol}, 1$ eq. $) \mathrm{UF}_{4}$ were placed in a reaction flask under argon atmosphere. After cooling to $195 \mathrm{~K}$ ca $10 \mathrm{ml} \mathrm{NH}_{3}$ were added to the reaction mixture resulting in a clear yellow solution and a green solid residue. Yellow single crystals of the title compound were obtained during storage at $233 \mathrm{~K}$ and were selected under cold perfluoroether oil (Kottke \& Stalke, 1993). Additionally, emerald green crystals of $\left[\mathrm{UF}_{4}\left(\mathrm{NH}_{3}\right)_{4}\right] \cdot \mathrm{NH}_{3}$ were observed (Kraus \& Baer, 2009) next to colourless crystals of $\mathrm{CsNO}_{3}$, both evidenced by determination of their unit-cell parameters.

\section{Refinement}

Crystal data, data collection and structure refinement details are summarized in Table 2. The structure was solved by the
Table 2

Experimental details.

\begin{tabular}{|c|c|}
\hline \multicolumn{2}{|l|}{ Crystal data } \\
\hline Chemical formula & {$\left[\mathrm{U}\left(\mathrm{NH}_{3}\right)_{5}\right] \mathrm{NO}_{3} \cdot \mathrm{NH}_{3}$} \\
\hline$M_{\mathrm{r}}$ & 434.24 \\
\hline Crystal system, space group & Orthorhombic, $\mathrm{Pbca}$ \\
\hline Temperature $(\mathrm{K})$ & 123 \\
\hline$a, b, c(\AA)$ & $15.7497(2), 7.7375(1), 18.8126(2)$ \\
\hline$V\left(\AA^{3}\right)$ & $2292.57(5)$ \\
\hline$Z$ & 8 \\
\hline Radiation type & Mo $K \alpha$ \\
\hline$\mu\left(\mathrm{mm}^{-1}\right)$ & 14.17 \\
\hline Crystal size $(\mathrm{mm})$ & $0.24 \times 0.21 \times 0.04$ \\
\hline
\end{tabular}

Data collection

Diffractometer

Absorption correction

$T_{\min }, T_{\max }$

No. of measured, independent and observed $[I>2 \sigma(I)]$ reflections

$R_{\text {int }}$

$(\sin \theta / \lambda)_{\max }\left(\AA^{-1}\right)$

Oxford Diffraction Xcalibur3

Multi-scan (CrysAlis RED; Oxford Diffraction, 2009)

$0.166,1.000$

$88079,6635,5051$

0.045

0.892

Refinement

$R\left[F^{2}>2 \sigma\left(F^{2}\right)\right], w R\left(F^{2}\right), S$

No. of reflections

No. of parameters

$\mathrm{H}$-atom treatment

$0.033,0.090,1.04$
6635
136
$\mathrm{H}$ atoms treated by a mixture of
$\quad$ independent and constrained
$\quad$ refinement
$5.60,-3.79$

$\Delta \rho_{\max }, \Delta \rho_{\min }\left(\mathrm{e} \AA^{-3}\right)$

$5.60,-3.79$

Computer programs: CrysAlis $C C D$ and CrysAlis RED (Oxford Diffraction, 2009), SHELXL97 (Sheldrick, 2008), SHELXL2014 (Sheldrick, 2015), SHELXLE (Hübschle et al., 2011), DIAMOND (Brandenburg, 2012) and publCIF (Westrip, 2010).

heavy-atom method and all other atoms were located from difference Fourier maps. In case of the hydrogen atoms of nitrogen atoms $\mathrm{N} 1-\mathrm{N} 5$, their positions were refined using a riding model with $\mathrm{N}-\mathrm{H}=0.91 \AA$ and $U_{\text {eq }}(\mathrm{H})=1.5 U_{\text {iso }}(\mathrm{N})$. The hydrogen atoms of the ammonia molecule of crystallization were refined freely. The maximum and minimum residual electron densities are located close to the $\mathrm{U}$ atom at distances of 0.58 and $0.04 \AA$, respectively.

\section{Acknowledgements}

FK thanks the Deutsche Forschungsgemeinschaft for his Heisenberg professorship. PW would like to thank the Deutsche Forschungsgemeinschaft for financial support during his $\mathrm{PhD}$ thesis.

\section{References}

Arnold, P. L., Love, J. B. \& Patel, D. (2009). Coord. Chem. Rev. 253, 1973-1978.

Barclay, G. A., Sabine, T. M. \& Taylor, J. C. (1965). Acta Cryst. 19, 205-209.

Baur, W. H. (1981). Interatomic Distance Predictions for Computer Simulation of Crystal Structures, in Structure and Bonding in Crystals, Vol. II, p. 31 ff, edited by M. O'Keeffe \& M. Navrotsky. New York: Academic Press.

Berthet, J.-C., Nierlich, M. \& Ephritikhine, M. (2003). Angew. Chem. Int. Ed. 42, 1952-1954.

Brandenburg, K. (2012). DIAMOND. Crystal Impact GbR, Bonn, Germany.

Hayton, T. W. \& Wu, G. (2008). J. Am. Chem. Soc. 130, 2005-2014. 
Hübschle, C. B., Sheldrick, G. M. \& Dittrich, B. (2011). J. Appl. Cryst. 44, 1281-1284.

Jouffret, L. J., Krivovichev, S. V. \& Burns, P. C. (2011). Z. Anorg. Allg. Chem. 637, 1475-1480.

Kottke, T. \& Stalke, D. (1993). J. Appl. Cryst. 26, 615-619.

Kraus, F. \& Baer, S. A. (2009). Chem. Eur. J. 15, 8269-8274.

Kraus, K. A., Nelson, F. \& Johnson, G. L. (1949). J. Am. Chem. Soc. 71, 2510-2517.

Krivovichev, S. V. \& Burns, P. C. (2004). Radiochemistry, 46, 16-19.

Malcic, S. S. \& Ljubica, L. M. (1961). Bull. Boris Kidric. Inst. Nucl. Sci. 11, 135-139.

Oxford Diffraction (2009). CrysAlis CCD and CrysAlis RED. Oxford Diffraction Ltd, Abingdon, England.
Sheldrick, G. M. (2008). Acta Cryst. A64, 112-122.

Sheldrick, G. M. (2015). Acta Cryst. C71, 3-8.

Westrip, S. P. (2010). J. Appl. Cryst. 43, 920-925.

Woidy, P., Bühl, M. \& Kraus, F. (2015b). Dalton Trans. 44, 73327337.

Woidy, P., Karttunen, A. J. \& Kraus, F. (2012). Z. Anorg. Allg. Chem. 638, 2044-2052.

Woidy, P., Karttunen, A. J., Widenmeyer, M., Niewa, R. \& Kraus, F. (2015a). Chem. Eur. J. 21, 3290-3303.

Zalkin, A., Templeton, L. K. \& Templeton, D. H. (1989). Acta Cryst. C45, 810-811.

Zemann, J. (1981). Fortschr. Mineral. 59, 95-116. 


\section{supporting information}

Acta Cryst. (2016). E72, 1710-1713 [https://doi.org/10.1107/S2056989016016480]

\section{Crystal structure of $\left[\mathrm{UO}_{2}\left(\mathrm{NH}_{3}\right)_{5}\right] \mathrm{NO}_{3} \cdot \mathrm{NH}_{3}$}

\section{Patrick Woidy and Florian Kraus}

\section{Computing details}

Data collection: CrysAlis CCD (Oxford Diffraction, 2009); cell refinement: CrysAlis RED (Oxford Diffraction, 2009); data reduction: CrysAlis RED (Oxford Diffraction, 2009); program(s) used to solve structure: SHELXL97 (Sheldrick, 2008); program(s) used to refine structure: SHELXL2014 (Sheldrick, 2015) and SHELXLE (Hübschle et al., 2011); molecular graphics: DIAMOND (Brandenburg, 2012); software used to prepare material for publication: publCIF

(Westrip, 2010).

Pentaammine dioxide uranium(V) nitrate ammonia monosolvate

\section{Crystal data}

$\left[\mathrm{U}\left(\mathrm{NH}_{3}\right)_{5}\right] \mathrm{NO}_{3} \cdot \mathrm{NH}_{3}$

$M_{r}=434.24$

Orthorhombic, $P b c a$

$a=15.7497(2) \AA$

$b=7.7375(1) \AA$

$c=18.8126(2) \AA$

$V=2292.57(5) \AA^{3}$

$Z=8$

$F(000)=1592$

\section{Data collection}

Oxford Diffraction Xcalibur3 diffractometer

Radiation source: Enhance (Mo) X-ray Source

Graphite monochromator

Detector resolution: 16.0238 pixels $\mathrm{mm}^{-1}$

phi- and $\omega$-rotation scans

Absorption correction: multi-scan

(CrysAlis RED; Oxford Diffraction, 2009)

$T_{\min }=0.166, T_{\max }=1.000$

\section{Refinement}

Refinement on $F^{2}$

Least-squares matrix: full

$R\left[F^{2}>2 \sigma\left(F^{2}\right)\right]=0.033$

$w R\left(F^{2}\right)=0.090$

$S=1.04$

6635 reflections

136 parameters

0 restraints

Primary atom site location: heavy-atom method
$D_{\mathrm{x}}=2.516 \mathrm{Mg} \mathrm{m}^{-3}$

Melting point: not measured $\mathrm{K}$

Mo $K \alpha$ radiation, $\lambda=0.71073 \AA$

Cell parameters from 44787 reflections

$\theta=2.8-39.2^{\circ}$

$\mu=14.17 \mathrm{~mm}^{-1}$

$T=123 \mathrm{~K}$

Plate, colourless

$0.24 \times 0.21 \times 0.04 \mathrm{~mm}$

88079 measured reflections

6635 independent reflections

5051 reflections with $I>2 \sigma(I)$

$R_{\text {int }}=0.045$

$\theta_{\max }=39.3^{\circ}, \theta_{\min }=3.1^{\circ}$

$h=-27 \rightarrow 27$

$k=-8 \rightarrow 13$

$l=-32 \rightarrow 32$

Secondary atom site location: difference Fourier map

Hydrogen site location: mixed

$\mathrm{H}$ atoms treated by a mixture of independent and constrained refinement

$w=1 /\left[\sigma^{2}\left(F_{\mathrm{o}}^{2}\right)+(0.0532 P)^{2}+3.9914 P\right]$

where $P=\left(F_{\mathrm{o}}{ }^{2}+2 F_{\mathrm{c}}{ }^{2}\right) / 3$

$(\Delta / \sigma)_{\max }=0.003$

$\Delta \rho_{\max }=5.60 \mathrm{e} \AA^{-3}$

$\Delta \rho_{\min }=-3.79 \mathrm{e}^{-3}$ 
Extinction correction: SHELXL2014 (Sheldrick, 2015),

$\mathrm{Fc}^{*}=\mathrm{kFc}\left[1+0.001 \times \mathrm{Fc}^{2} \lambda^{3} / \sin (2 \theta)\right]^{-1 / 4}$

Extinction coefficient: 0.00070 (7)

\section{Special details}

Geometry. All esds (except the esd in the dihedral angle between two 1.s. planes) are estimated using the full covariance matrix. The cell esds are taken into account individually in the estimation of esds in distances, angles and torsion angles; correlations between esds in cell parameters are only used when they are defined by crystal symmetry. An approximate (isotropic) treatment of cell esds is used for estimating esds involving l.s. planes.

Fractional atomic coordinates and isotropic or equivalent isotropic displacement parameters $\left(\AA^{2}\right)$

\begin{tabular}{|c|c|c|c|c|}
\hline & $x$ & $y$ & $z$ & $U_{\text {iso }} * / U_{\text {eq }}$ \\
\hline U1 & $0.11450(2)$ & $0.22849(2)$ & $0.80178(2)$ & $0.00916(4)$ \\
\hline $\mathrm{O} 1$ & $0.11836(14)$ & $-0.0112(4)$ & $0.79457(13)$ & $0.0145(4)$ \\
\hline $\mathrm{O} 2$ & $0.10583(16)$ & $0.4688(4)$ & $0.80629(15)$ & $0.0180(5)$ \\
\hline N1 & $0.26344(17)$ & $0.2174(4)$ & $0.86194(16)$ & $0.0140(5)$ \\
\hline $\mathrm{H} 1 \mathrm{~A}$ & 0.257174 & 0.197530 & 0.909327 & $0.021 *$ \\
\hline H1B & 0.290520 & 0.320039 & 0.855278 & $0.021 *$ \\
\hline $\mathrm{H} 1 \mathrm{C}$ & 0.294646 & 0.130809 & 0.842227 & $0.021^{*}$ \\
\hline $\mathrm{N} 2$ & $0.0977(2)$ & $0.2180(5)$ & $0.93878(17)$ & $0.0182(6)$ \\
\hline $\mathrm{H} 2 \mathrm{~A}$ & 0.111922 & 0.110928 & 0.954839 & $0.027 *$ \\
\hline $\mathrm{H} 2 \mathrm{~B}$ & 0.042857 & 0.241370 & 0.950589 & $0.027^{*}$ \\
\hline $\mathrm{H} 2 \mathrm{C}$ & 0.132433 & 0.298114 & 0.959019 & $0.027 *$ \\
\hline N3 & $-0.04957(19)$ & $0.2180(4)$ & $0.81645(19)$ & $0.0185(6)$ \\
\hline $\mathrm{H} 3 \mathrm{~A}$ & -0.070849 & 0.128341 & 0.790693 & $0.028^{*}$ \\
\hline H3B & -0.072525 & 0.318832 & 0.800654 & $0.028^{*}$ \\
\hline $\mathrm{H} 3 \mathrm{C}$ & -0.062761 & 0.203054 & 0.863146 & $0.028^{*}$ \\
\hline N4 & $0.0394(2)$ & $0.2335(4)$ & $0.67697(19)$ & $0.0188(6)$ \\
\hline $\mathrm{H} 4 \mathrm{~A}$ & 0.003321 & 0.325238 & 0.674759 & $0.028 *$ \\
\hline H4B & 0.009622 & 0.133855 & 0.670795 & $0.028^{*}$ \\
\hline $\mathrm{H} 4 \mathrm{C}$ & 0.079243 & 0.243219 & 0.642176 & $0.028^{*}$ \\
\hline N5 & $0.2334(2)$ & $0.2756(5)$ & $0.70995(18)$ & $0.0190(6)$ \\
\hline $\mathrm{H} 5 \mathrm{~A}$ & 0.214265 & 0.346174 & 0.674728 & $0.029 *$ \\
\hline H5B & 0.248928 & 0.172025 & 0.691072 & $0.029^{*}$ \\
\hline $\mathrm{H} 5 \mathrm{C}$ & 0.278944 & 0.325155 & 0.731585 & $0.029^{*}$ \\
\hline N6 & $-0.0713(3)$ & $0.3021(8)$ & $1.0069(3)$ & $0.0327(9)$ \\
\hline H6A & $-0.110(4)$ & $0.227(8)$ & $1.013(4)$ & $0.033(19)^{*}$ \\
\hline H6B & $-0.095(4)$ & $0.381(10)$ & $0.986(4)$ & $0.05(2)^{*}$ \\
\hline $\mathrm{H} 6 \mathrm{C}$ & $-0.056(6)$ & $0.312(12)$ & $1.037(5)$ & $0.06(3)^{*}$ \\
\hline N7 & $0.2550(2)$ & 0.0309 (4) & $0.55493(17)$ & $0.0204(6)$ \\
\hline $\mathrm{O} 3$ & $0.1861(2)$ & $0.0340(5)$ & $0.58716(17)$ & $0.0320(7)$ \\
\hline $\mathrm{O} 4$ & 0.25705 (19) & $0.0485(4)$ & $0.48871(15)$ & $0.0266(6)$ \\
\hline $\mathrm{O} 5$ & $0.3231(2)$ & $0.0085(5)$ & $0.58813(17)$ & $0.0311(7)$ \\
\hline
\end{tabular}


Atomic displacement parameters $\left(\AA^{2}\right)$

\begin{tabular}{lllllll}
\hline & $U^{11}$ & $U^{22}$ & $U^{33}$ & $U^{12}$ & $U^{13}$ & $U^{23}$ \\
\hline $\mathrm{U} 1$ & $0.00711(5)$ & $0.00894(6)$ & $0.01145(5)$ & $0.00013(3)$ & $-0.00104(3)$ & $-0.00027(3)$ \\
$\mathrm{O} 1$ & $0.0137(10)$ & $0.0142(12)$ & $0.0157(11)$ & $-0.0007(8)$ & $-0.0016(8)$ & $-0.0011(8)$ \\
O2 & $0.0176(11)$ & $0.0096(11)$ & $0.0267(14)$ & $0.0021(8)$ & $-0.0032(9)$ & $-0.0019(9)$ \\
N1 & $0.0093(10)$ & $0.0178(14)$ & $0.0147(11)$ & $0.0020(9)$ & $-0.0012(8)$ & $-0.0006(10)$ \\
N2 & $0.0140(11)$ & $0.0250(16)$ & $0.0156(12)$ & $0.0012(10)$ & $0.0010(10)$ & $-0.0005(11)$ \\
N3 & $0.0111(11)$ & $0.0222(16)$ & $0.0221(14)$ & $-0.0006(10)$ & $-0.0004(10)$ & $0.0003(11)$ \\
N4 & $0.0184(13)$ & $0.0214(15)$ & $0.0167(12)$ & $-0.0025(11)$ & $-0.0054(11)$ & $0.0006(11)$ \\
N5 & $0.0167(13)$ & $0.0230(16)$ & $0.0172(13)$ & $-0.0017(11)$ & $0.0026(10)$ & $-0.0014(11)$ \\
N6 & $0.0188(15)$ & $0.049(3)$ & $0.030(2)$ & $0.0031(17)$ & $0.0034(15)$ & $0.002(2)$ \\
N7 & $0.0263(16)$ & $0.0193(15)$ & $0.0158(13)$ & $-0.0001(12)$ & $-0.0030(11)$ & $-0.0010(11)$ \\
O3 & $0.0275(14)$ & $0.044(2)$ & $0.0242(15)$ & $0.0026(14)$ & $0.0024(12)$ & $-0.0076(14)$ \\
O4 & $0.0347(16)$ & $0.0330(17)$ & $0.0121(11)$ & $0.0024(13)$ & $-0.0015(11)$ & $0.0020(11)$ \\
O5 & $0.0269(14)$ & $0.0409(19)$ & $0.0255(15)$ & $-0.0051(13)$ & $-0.0095(12)$ & $0.0083(14)$ \\
& & & & & &
\end{tabular}

Geometric parameters $\left(\AA,{ }^{\circ}\right)$

\begin{tabular}{|c|c|c|c|}
\hline $\mathrm{U} 1-\mathrm{O} 1$ & $1.861(3)$ & $\mathrm{N} 3-\mathrm{H} 3 \mathrm{~B}$ & 0.9100 \\
\hline $\mathrm{U} 1-\mathrm{O} 2$ & $1.867(3)$ & $\mathrm{N} 3-\mathrm{H} 3 \mathrm{C}$ & 0.9100 \\
\hline $\mathrm{U} 1-\mathrm{N} 5$ & $2.573(3)$ & $\mathrm{N} 4-\mathrm{H} 4 \mathrm{~A}$ & 0.9100 \\
\hline $\mathrm{U} 1-\mathrm{N} 2$ & $2.592(3)$ & $\mathrm{N} 4-\mathrm{H} 4 \mathrm{~B}$ & 0.9100 \\
\hline $\mathrm{U} 1-\mathrm{N} 3$ & $2.600(3)$ & $\mathrm{N} 4-\mathrm{H} 4 \mathrm{C}$ & 0.9100 \\
\hline $\mathrm{U} 1-\mathrm{N} 1$ & $2.606(3)$ & N5-H5A & 0.9100 \\
\hline $\mathrm{U} 1-\mathrm{N} 4$ & $2.629(3)$ & N5-H5B & 0.9100 \\
\hline $\mathrm{N} 1-\mathrm{H} 1 \mathrm{~A}$ & 0.9100 & $\mathrm{~N} 5-\mathrm{H} 5 \mathrm{C}$ & 0.9100 \\
\hline N1-H1B & 0.9100 & N6- $\mathrm{H} 6 \mathrm{~A}$ & $0.86(7)$ \\
\hline $\mathrm{N} 1-\mathrm{H} 1 \mathrm{C}$ & 0.9100 & N6- $\mathrm{H} 6 \mathrm{~B}$ & $0.81(8)$ \\
\hline $\mathrm{N} 2-\mathrm{H} 2 \mathrm{~A}$ & 0.9100 & $\mathrm{~N} 6-\mathrm{H} 6 \mathrm{C}$ & $0.63(9)$ \\
\hline $\mathrm{N} 2-\mathrm{H} 2 \mathrm{~B}$ & 0.9100 & $\mathrm{~N} 7-\mathrm{O} 3$ & $1.242(5)$ \\
\hline $\mathrm{N} 2-\mathrm{H} 2 \mathrm{C}$ & 0.9100 & $\mathrm{~N} 7-\mathrm{O} 5$ & $1.253(4)$ \\
\hline $\mathrm{N} 3-\mathrm{H} 3 \mathrm{~A}$ & 0.9100 & $\mathrm{~N} 7-\mathrm{O} 4$ & $1.254(4)$ \\
\hline $\mathrm{O} 1-\mathrm{U} 1-\mathrm{O} 2$ & $177.20(11)$ & $\mathrm{H} 2 \mathrm{~A}-\mathrm{N} 2-\mathrm{H} 2 \mathrm{~B}$ & 109.5 \\
\hline $\mathrm{O} 1-\mathrm{U} 1-\mathrm{N} 5$ & $93.92(11)$ & $\mathrm{U} 1-\mathrm{N} 2-\mathrm{H} 2 \mathrm{C}$ & 109.5 \\
\hline $\mathrm{O} 2-\mathrm{U} 1-\mathrm{N} 5$ & $86.72(12)$ & $\mathrm{H} 2 \mathrm{~A}-\mathrm{N} 2-\mathrm{H} 2 \mathrm{C}$ & 109.5 \\
\hline $\mathrm{O} 1-\mathrm{U} 1-\mathrm{N} 2$ & $92.56(11)$ & $\mathrm{H} 2 \mathrm{~B}-\mathrm{N} 2-\mathrm{H} 2 \mathrm{C}$ & 109.5 \\
\hline $\mathrm{O} 2-\mathrm{U} 1-\mathrm{N} 2$ & $88.76(12)$ & $\mathrm{U} 1-\mathrm{N} 3-\mathrm{H} 3 \mathrm{~A}$ & 109.5 \\
\hline $\mathrm{N} 5-\mathrm{U} 1-\mathrm{N} 2$ & $138.26(11)$ & $\mathrm{U} 1-\mathrm{N} 3-\mathrm{H} 3 \mathrm{~B}$ & 109.5 \\
\hline $\mathrm{O} 1-\mathrm{U} 1-\mathrm{N} 3$ & $90.52(11)$ & $\mathrm{H} 3 \mathrm{~A}-\mathrm{N} 3-\mathrm{H} 3 \mathrm{~B}$ & 109.5 \\
\hline $\mathrm{O} 2-\mathrm{U} 1-\mathrm{N} 3$ & $87.34(11)$ & $\mathrm{U} 1-\mathrm{N} 3-\mathrm{H} 3 \mathrm{C}$ & 109.5 \\
\hline $\mathrm{N} 5-\mathrm{U} 1-\mathrm{N} 3$ & $143.01(11)$ & $\mathrm{H} 3 \mathrm{~A}-\mathrm{N} 3-\mathrm{H} 3 \mathrm{C}$ & 109.5 \\
\hline $\mathrm{N} 2-\mathrm{U} 1-\mathrm{N} 3$ & $78.00(11)$ & $\mathrm{H} 3 \mathrm{~B}-\mathrm{N} 3-\mathrm{H} 3 \mathrm{C}$ & 109.5 \\
\hline $\mathrm{O} 1-\mathrm{U} 1-\mathrm{N} 1$ & $88.25(10)$ & $\mathrm{U} 1-\mathrm{N} 4-\mathrm{H} 4 \mathrm{~A}$ & 109.5 \\
\hline $\mathrm{O} 2-\mathrm{U} 1-\mathrm{N} 1$ & $94.52(10)$ & $\mathrm{U} 1-\mathrm{N} 4-\mathrm{H} 4 \mathrm{~B}$ & 109.5 \\
\hline $\mathrm{N} 5-\mathrm{U} 1-\mathrm{N} 1$ & $68.98(10)$ & $\mathrm{H} 4 \mathrm{~A}-\mathrm{N} 4-\mathrm{H} 4 \mathrm{~B}$ & 109.5 \\
\hline $\mathrm{N} 2-\mathrm{U} 1-\mathrm{N} 1$ & $70.06(10)$ & $\mathrm{U} 1-\mathrm{N} 4-\mathrm{H} 4 \mathrm{C}$ & 109.5 \\
\hline
\end{tabular}




$\begin{array}{llll}\text { N3-U1-N1 } & 147.94(10) & \mathrm{H} 4 \mathrm{~A}-\mathrm{N} 4-\mathrm{H} 4 \mathrm{C} & 109.5 \\ \text { O1-U1-N4 } & 87.96(11) & \mathrm{H} 4 \mathrm{~B}-\mathrm{N} 4-\mathrm{H} 4 \mathrm{C} & 109.5 \\ \text { O2-U1-N4 } & 89.59(11) & \mathrm{U} 1-\mathrm{N} 5-\mathrm{H} 5 \mathrm{~A} & 109.5 \\ \text { N5-U1-N4 } & 74.08(11) & \mathrm{U} 1-\mathrm{N} 5-\mathrm{H} 5 \mathrm{~B} & 109.5 \\ \text { N2-U1-N4 } & 147.40(11) & \mathrm{H} 5 \mathrm{~A}-\mathrm{N} 5-\mathrm{H} 5 \mathrm{~B} & 109.5 \\ \text { N3-U1-N4 } & 69.40(11) & \mathrm{U} 1-\mathrm{N} 5-\mathrm{H} 5 \mathrm{C} & 109.5 \\ \text { N1-U1-N4 } & 142.49(10) & \mathrm{H} 5 \mathrm{~A}-\mathrm{N} 5-\mathrm{H} 5 \mathrm{C} & 109.5 \\ \mathrm{U} 1-\mathrm{N} 1-\mathrm{H} 1 \mathrm{~A} & 109.5 & \mathrm{H} 5 \mathrm{~B}-\mathrm{N} 5-\mathrm{H} 5 \mathrm{C} & 109.5 \\ \mathrm{U} 1-\mathrm{N} 1-\mathrm{H} 1 \mathrm{~B} & 109.5 & \mathrm{H} 6 \mathrm{~A}-\mathrm{N} 6-\mathrm{H} 6 \mathrm{~B} & 104(7) \\ \mathrm{H} 1 \mathrm{~A}-\mathrm{N} 1-\mathrm{H} 1 \mathrm{~B} & 109.5 & \mathrm{H} 6 \mathrm{~A}-\mathrm{N} 6-\mathrm{H} 6 \mathrm{C} & 103(10) \\ \mathrm{U} 1-\mathrm{N} 1-\mathrm{H} 1 \mathrm{C} & 109.5 & \mathrm{H} 6 \mathrm{~B}-\mathrm{N} 6-\mathrm{H} 6 \mathrm{C} & 121(10) \\ \mathrm{H} 1 \mathrm{~A}-\mathrm{N} 1-\mathrm{H} 1 \mathrm{C} & 109.5 & \mathrm{O} 3-\mathrm{N} 7-\mathrm{O} 4 & 120.4(3) \\ \mathrm{H} 1 \mathrm{~B}-\mathrm{N} 1-\mathrm{H} 1 \mathrm{C} & 109.5 & \mathrm{O} 5-\mathrm{N} 7-\mathrm{O} 4 & 120.4(3) \\ \mathrm{U} 1-\mathrm{N} 2-\mathrm{H} 2 \mathrm{~A} & 109.5 & & 119.2(3) \\ \mathrm{U} 1-\mathrm{N} 2-\mathrm{H} 2 \mathrm{~B} & 109.5 & & \end{array}$

Hydrogen-bond geometry $\left(\AA,{ }^{\circ}\right)$

\begin{tabular}{|c|c|c|c|c|}
\hline$D-\mathrm{H} \cdots A$ & $D-\mathrm{H}$ & $\mathrm{H} \cdots A$ & $D \cdots A$ & $D-\mathrm{H} \cdots A$ \\
\hline $\mathrm{N} 1-\mathrm{H} 1 A \cdots \mathrm{O} 4^{\mathrm{i}}$ & 0.91 & 2.43 & $3.166(4)$ & 138 \\
\hline $\mathrm{N} 1-\mathrm{H} 1 A \cdots \mathrm{O} 4^{\mathrm{ii}}$ & 0.91 & 2.47 & $2.996(4)$ & 117 \\
\hline $\mathrm{N} 1-\mathrm{H} 1 B \cdots \mathrm{O} 1^{\mathrm{iii}}$ & 0.91 & 2.25 & $3.079(4)$ & 151 \\
\hline $\mathrm{N} 1-\mathrm{H} 1 C^{\prime} \cdots \mathrm{O} 2^{\mathrm{iv}}$ & 0.91 & 2.12 & $3.006(4)$ & 165 \\
\hline $\mathrm{N} 2-\mathrm{H} 2 A \cdots \mathrm{O} 4^{\mathrm{i}}$ & 0.91 & 2.49 & $3.220(5)$ & 138 \\
\hline $\mathrm{N} 2-\mathrm{H} 2 B \cdots \mathrm{N} 6$ & 0.91 & 2.14 & $3.024(5)$ & 164 \\
\hline $\mathrm{N} 2-\mathrm{H} 2 C \cdots \mathrm{O} 4^{\mathrm{ii}}$ & 0.91 & 2.36 & $3.232(5)$ & 160 \\
\hline $\mathrm{N} 3-\mathrm{H} 3 A \cdots \mathrm{O} 2^{\mathrm{v}}$ & 0.91 & 2.27 & $3.136(5)$ & 159 \\
\hline $\mathrm{N} 3-\mathrm{H} 3 B^{\cdots} \mathrm{O} 1^{\mathrm{vi}}$ & 0.91 & 2.34 & $3.151(4)$ & 149 \\
\hline $\mathrm{N} 3-\mathrm{H} 3 C^{-\cdots} \mathrm{O} 5^{\mathrm{vii}}$ & 0.91 & 2.52 & $3.142(5)$ & 126 \\
\hline $\mathrm{N} 4-\mathrm{H} 4 A^{\cdots} \mathrm{O} 1^{\mathrm{vi}}$ & 0.91 & 2.37 & $3.219(4)$ & 156 \\
\hline $\mathrm{N} 4-\mathrm{H} 4 B \cdots \mathrm{O} 2^{\mathrm{v}}$ & 0.91 & 2.26 & $3.086(4)$ & 150 \\
\hline $\mathrm{N} 4-\mathrm{H} 4 C_{\cdots} \cdots \mathrm{O} 3$ & 0.91 & 2.55 & $3.253(5)$ & 134 \\
\hline $\mathrm{N} 5-\mathrm{H} 5 A \cdots \mathrm{O} 5^{\mathrm{iii}}$ & 0.91 & 2.14 & $3.048(5)$ & 176 \\
\hline $\mathrm{N} 5-\mathrm{H} 5 B \cdots \mathrm{O} 3$ & 0.91 & 2.44 & $3.063(5)$ & 126 \\
\hline $\mathrm{N} 5-\mathrm{H} 5 B \cdots \mathrm{O} 5$ & 0.91 & 2.59 & $3.394(5)$ & 147 \\
\hline $\mathrm{N} 5-\mathrm{H} 5 C \cdots \mathrm{O} 1^{\mathrm{iii}}$ & 0.91 & 2.37 & $3.273(4)$ & 171 \\
\hline $\mathrm{N} 6-\mathrm{H} 6 A \cdots \mathrm{O} 4^{\mathrm{vii}}$ & $0.86(7)$ & $2.50(7)$ & $3.342(6)$ & $167(7)$ \\
\hline $\mathrm{N} 6-\mathrm{H} 6 B \cdots \mathrm{O} 3^{\mathrm{vi}}$ & $0.81(8)$ & $2.32(8)$ & $3.102(6)$ & $162(7)$ \\
\hline
\end{tabular}

Symmetry codes: (i) $-x+1 / 2,-y, z+1 / 2$; (ii) $x,-y+1 / 2, z+1 / 2$; (iii) $-x+1 / 2, y+1 / 2, z$; (iv) $-x+1 / 2, y-1 / 2, z$; (v) $-x, y-1 / 2,-z+3 / 2$; (vi) $-x, y+1 / 2,-z+3 / 2$; (vii) $x-1 / 2, y,-z+3 / 2$. 